\title{
Data Interface Design of Digital City Management System
}

\author{
http://dx.doi.org/10.3991/ijoe.v9iS7.3196 \\ Changhui Yang \\ School of Business, Zhengzhou University, Henan Zhengzhou, China
}

\begin{abstract}
It is just because of the application of information technology that improve the level and efficiency of city management as well as the management quality of government public service. In this paper, the general structure of city management system and its subsystems have been analyzed, and then discusses how to design the data interface between the related subsystem of digital city management system, it will ensure the expedite transfer between the related subsystem, and offer the timely and exact data for city managers, that will be benefit to advance decision-making and quick response of emergency for city managers.
\end{abstract}

Index Terms-Digital City Management System. Data Interface. Data Transfer. System Structure.

\section{INTRODUCTION}

City management is a necessary requirement for sustainable development, which is also an important measure for city economic and social development, meanwhile, it guarantees the process of urbanization and the establishment of the modern city. It is conducive to implement the overall city planning and to enhance the authority of the city planning to strengthen the city management; Also, it favors the normal function of city infrastructure and benefit of city investment; Additionally, it makes for the improvement of the city environment, city image and degree of opening to the outside world; Apart from these, it helps enhance the comprehensive functions of cities, and promote the sustainable development of city economics and society. To establish pilots before building digital city management system can accumulate valuable experience for the comprehensive construction, which can also guarantee the whole work.

The construction of digital city runs throughout the whole process of city planning, city construction, city management and service. With the construction of digital city and the use of information technology, it helps promote the transformation of government functions and the innovation of management. The innovation of system leads to the improvement of the government management and service, and then, achieves efficient service. On the viewpoint of development, without the building new mode of city management and planning and managing city according to human-oriented thought, it can neither get to a new breakthrough for city management, nor create a favorable condition for the development and stability of city reform. With the system construction, the work that promotes city management to get to the target of initiative, accuracy, speediness and unification, integrates and optimizes the governmental information resources and the government database groups, sets up city management system covering the whole period and the whole region. To establish pilots before building digital city management system can accumulate valuable experience for the comprehensive construction, which can also guarantee the whole work.

\section{SYSTEM STRUCTURE OF DIGITAL CITY MANAGEMENT SYSTEM}

\section{A. System structure of digital city management system}

According to the "two axis" city management mode, the digital city management system includes city management monitoring and evaluation center(i.e. supervision center of the city management), as well as the command, scheduling, coordinating center(i.e. command center of the city management).The purpose of such a setting is to separate supervision from command, each fulfills its own duties. In order to realize the new city management system, the two axis of supervision and command should be connected seamlessly and work efficiently in a coordinate manner through the platform of the digital city management. Generally speaking, the digital city management system can be divided into 10 subsystems, which are shown in figure1.

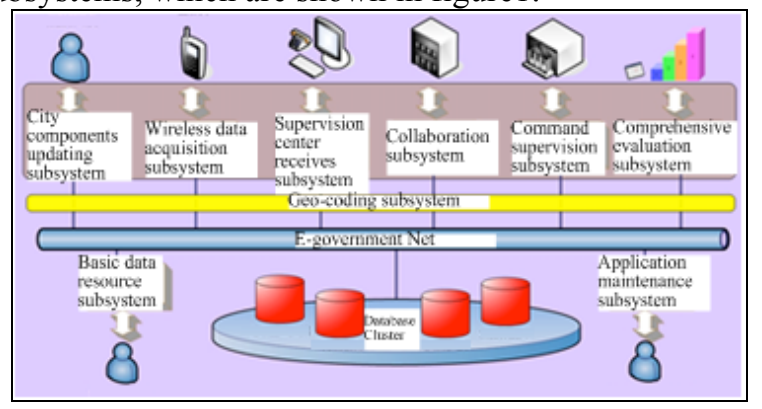

Fig. 1 System structure of digital city management system

\section{B. The subsystems of city management system}

1. Subsystem of wireless data acquisition

This subsystem is mainly used for the supervisor to report the information about the city management issues during their inspection in their own management unit mesh. The system based on mobile devices, using 
wireless communication network data transmission technology, relying the city components and events classification coding system and geo-coding system, completes the transmission of city management issues information in words, images, sounds and location information real-timely.

2. Subsystem of supervision center receives

This subsystem is designed for the supervision center of city management, employing personnel as supervision center operators. The information submitted by the "subsystem of wireless data acquisition" is passed to the working platform of the operators through the information service engines. And then, the operators receive, process and feedback various problems through the system to finish the collection, processing and filing of information. All the work provides collection and filing service of the city management issues for "the subsystem of collaboration" and ensures that problematical information can be accepted timely and accurately, and transferred to the command center.

3. Subsystem of collaboration

This subsystem, based on Browser/Server architecture, applying workflow and WebGIS technology, completes the specific transactions and information inquiries of each city management business through the browser. The subsystem of collaboration serves for the supervision center, the command center, various professional sectors, as well as leaders at all levels. The subsystem provides a workflow-based GIS for collaborative management, workflow processing, and supervision, it also offers the tools to share and query types of information resources of city management for all kinds of users. Additional, based on different authority, it edits and queries geographic information, geoinformation, city management components (events) information, supervision information, and so on. This system has realized the synergy office, the information synchronization and the exchange of information.

4. Subsystem of city components updating online

According to the specification of components encoding, this subsystem provides specific access control modules to meet the needs of professional departments, who can update and maintain the data on the components through the IE browser.

5. Subsystem of command and supervision on largescreen

This subsystem services for supervision center and command center, illustrating exactly the overall situation about the related map information, file information and other detailed information of the city management on the large screen. Similarly, the system is able to query and present information of each community, supervisor and component and other individuals, and then get to hold of the overall situation of city management.

6. Subsystem of comprehensive evaluation for city management

For the performance quantification and comprehensive evaluation, this subsystem makes a comprehensive analysis and calculates estimates on the information of regions, departments and positions in the database groups, generating performance-based evaluation results in accordance with the process, the main responsibility, work performance, normal specification and other evaluation models built-in the system.

7. Subsystem of geo-coding

This subsystem furnishes the subsystems of wireless data acquisition, collaboration, supervision and command on large-screen and others with geo-coding service, playing a role of describing, inquiring, and matching address.

8. Subsystem of application and maintenance

Responsible for the configuration, maintenance and management the whole system, applying the instrumentalization theory, this subsystem completes the flexible configuration of city management information, such as organizational structure, workflow, input form, output form, the use of map, inquiry and statistics. It can be configured to form the corresponding resource information, business rules and the definition of data manipulation using kinds of tools and components supplied by the system.

9. Subsystem of basic data resource management

This subsystem is used to realize the management, maintenance and expansion of spatial data resource, moreover, configure the functions of displaying, querying, editing and calculating spatial data.

10. Subsystem of data-exchanging and sharing system platform

Some information technologies such as mature XML, SOAP and Web Service can be used to establish an information-exchange platform, which includes the data exchange engine, remote data transmission and standard of exchange information and other core modules.

\section{DESIGN RELEVANT SYSTEM INTERFACE OF DIGITAL CITY MANAGEMENT SYSTEM}

\section{A. Interface of wireless data transmission}

Wireless data transmission mainly refers to the data transmission between wireless data acquisition system and database service. Using wireless data collection tool, we can transmit the collected related information on event (component) including the types, relevant pictures, audio materials, location coordinates and other information of event (component) to the service by GPRS data transmission technology.

The data transmission between wireless terminal and service should support the HTTP hypertext transfer protocol and being able to achieve the information transmission in text, graphics, sound and image. Therefore, it should fit with the following requirements:

1. support HTTP hypertext transfer protocol;

2. support the technology of wireless data transmission;

3. support data encryption;

4. the speed rate of wireless actual communication can't be less than $30 \mathrm{Kbps}$;

5. maintain stable network communication 
quality; the success rate of the transmission of information must be more than $99.9 \%$.

6. concurrent users who exchange and transform data with the service should be able to guarantee the good running of system.

7. The time for one-time wireless data exchange and transmission is expected to be shorter than 30 seconds.

\section{B. Interface among the subsystems of wireless data acquisition, calling accepted and collaboration}

The subsystem of wireless data acquisition installed in the wireless data collection tool is mainly applied to collect the information of event (component) and send the results to the subsystem of calling accepted through a wireless network. After the results have been filed, it will get to subsystem of the collaboration. The subsystem of calling accepted and collaboration have the same server, so it is easy to exchange and retrieval the data. In addition to what have been mentioned, the subsystem of collaboration will transmit data with subsystem of wireless data acquisition, which mainly occurs when the files of the event (component) have been treated. It requires some supervisors to verify if the case scene has been correctly handled. Therefore, on some node of the process, the subsystem of collaboration has to send the subsystem of wireless data acquisition the verification instructions which can reflect the relevant information of event (component) and location information and so on in the subsystem of wireless data. After the on-site verification in accordance with the relevant information, supervisors feed back the results into the nodes of subsystem of collaboration.

The data needs to exchange real-timely is divided into two types: one is information of the event (component) sent to the subsystem of calling accepted by city supervisors through subsystem of wireless data acquisition, the other is task information sent to the city supervisors by working platform of calling s based on the need of tasks. Task information includes:

(1) information reported about the social work and information that needs to be confirmed by the city supervisors.

(2) information assigned task number by the calling center according to the incident reports of city supervisors.

(3) information fed back by city supervisors when they assist at the scene on the demand of calling center in accordance with the situation of handling of the incidents.

In the light of two types of information mentioned above, the information server is mainly concerned with two things: firstly, receive incident information sent by the subsystem of wireless data acquisition, read data from database and write it into database of city management platform, and send message to working platform of calling center. Secondly, receive task message from working platform of calling center, read data from database of city management platform and write it into the database of subsystem of wireless data acquisition, and send message to subsystem of wireless data acquisition. The message server plays a role as a data bridge between subsystem of wireless data acquisition and working platform of calling center, helping city supervisors and calling center to exchange message with each other real-timely.

Message server can also do some other things, such as, providing function of updating data initiatively, read database of subsystem of wireless data acquisition from time to time, adding new event (component) to the database of city management platform, sending event (component) message to working platform of calling center, noticing working platform of calling center that there have been new incident message to be dealt with.

\section{Interface among the subsystems of wireless data acquisition, collaboration and geo-coding}

The subsystem of geo-coding is composed of geocoding and geo-coded database. Assisted by geo-coding engines, the subsystems of wireless data acquisition and collaboration can complete the function of rapid positioning when they quickly navigate to the site of the incident through the address information.

Speeding and efficient positioning of associated components in the city management issues depends on the geo-positioning engine provided by subsystem of geocoding. Only when the received information quickly navigated on the map, the city management department can have a good understand of the location of the incident, the surrounding environment, traffic condition, and then have a command timely. Because of this, the geo-positioning engine of geo-coding should be fit with the following requirements:

1. Locate all the non-spatial socio-economic information collected and investigated (with the address) on the map exactly and establish the relationship between information of non-spatial and spatial accurately.

2. Lock the location of the incident quickly in the grid units of meters for the supervision center of the city management.

3. Site the arbitrary input address string on some position in the grid units of meters and provide the coordinate information of the location.

4. Generate geo-coding strings in standard quickly for any spatial location.

In short, digital city management makes the achievement of changing city management from the blind to the precise, from the manual to the informational.

\section{CONCLUSIONS}

It is the development trend of city management to manage the city using the information technology. City management system provides information timely and accurately for the government to manage the social, solves the problems like information lagged and management passive, improves the level and efficiency of city management as well as the management quality of government public service, helps enhance the comprehensive functions of cities, and promote the 


\section{SPECIAL FOCUS PAPER \\ DAta InTERFACE Design of Digital City MANAGEMENT SyStem}

sustainable development of city economics and society. In this paper, the general structure of city management system and have been analyzed, and then discusses how to design the data interface between the related subsystems of digital city management system, it will ensure the expedite transfer between the related subsystem, guarantees the data transmission between different subsystems timely and accurately, offers the efficient data for the city managers.

\section{ACKNOWLEDGMENT}

This paper was supported by NSFC (71272207, 40901071), 10YJC630326 (Humanity and Social Science Foundation of Ministry of Education).

\section{REFERENCES}

[1] Zhang Yunfan, Xu Yabin, Based on CTI technology call center design and realization, Liaoning engineering institute journal, February, 2006

[2] Jing Bo, Chen Ming, Guo Guanqing, Distributional and central unifies call center, Computer application, July, 2002

[3] Chen Wenhua, Huang Xiaoping, Chen Kai, Bai Yingcai, Based on VoIP technology virtual call center design and realization, Computer application and software, February, 2006
[4] Geurt, Jongbloed, Ger. Koole. Managing uncertainty in call centers using Poisson mixtures. Applied Stochastic Models in Business and Industry, 2001,17: 307-318. http://dx.doi.org/10.1002/asmb.444

[5] Ji Luping, Luo Kelu, Tan Hua, Based on three structures disperser -like call center system, Computer application, December, 2004

[6] Yang Changhui, Innovation System Framework of SME and Innovation Case Study, Proceedings of 2007 International Conference on Enterprise Engineering and Management Innovation, P969-972.

[7] Sun Xuemin, Yang Changhui, Constructing Service Call Center of Digital City Management System, Proceeding of 2nd International Conference on Intelligent Information Management Systems and Technology, 2007, VOL II, P307-309.

[8] Yang Changhui, Multi-Agent System of Evaluating and Selecting Supplier Basing on Quick Response Ability, Proceedings of the Sixth Wuhan International Conference on E-Business: Management Challenges in a Global World, Alfred University Press, 2007 (5), P1008-1012.

\section{AUTHOR}

Changhui Yang is with School of Business, Zhengzhou University, Henan Zhengzhou, China (yang0825@126.com)

Submitted 16 September 2013. Published as re-submitted by the author 22 October 2013 